NASA Technical Memorandum 104087

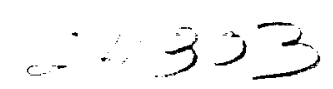

\title{
A METHODOLOGY FOR USING NONLINEAR AERODYNAMICS IN AEROSERVOELASTIC ANALYSIS AND DESIGN
}

WALTER A. SILVA

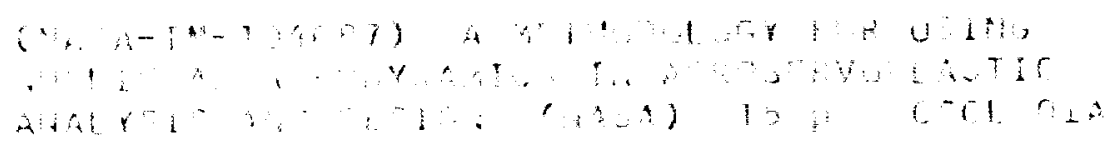





\title{
A METHODOLOGY FOR USING NONLINEAR AERODYNAMICS IN AEROSERVOELASTIC ANALYSIS AND DESIGN
}

\author{
Walter A. Silva* \\ Unsteady Aerodynamics Branch \\ NASA Langley Research Center \\ Hampton, VA 23665-5225
}

\begin{abstract}
A methodology is presented for using the VolterraWiener theory of nonlinear systems in aeroservoclastic (ASE) analyses and design. The theory is applied to the development of nonlinear aerodynamic response models that can be defined in state-space form and are, therefore, appropriate for use in modern control theory. The theory relies on the identification of nonlinear kernels that can be used to predict the response of a nonlinear system due to an arbitrary input. A numerical kernel identification technique, based on unit impulse responses, is presented and applied to a simple bilinear, single-input-singleoutput (SISO) system. The linear kernel (unit impulse response) and the nonlinear sccond-order kemel of the system are numerically-identified and compared with the exact, analytically-defined linear and second-order kernels. This kernel identification technique is then applied to the CAP-TSD (Computational Acroclasticity ProgramTransonic Small Disturbance) code for identification of the linear and second-order kernels of a NACA64A010 rectangular wing undergoing pitch at $M=0.5, M=0.85$ (transonic), and $\mathrm{M}=0.93$ (transonic). Results presented demonstrate the feasibility of this approach for use with nonlinear, unsteady aerodynamic responses.
\end{abstract}

\section{INTRODUCTION}

The subject of nonlinear unstcady aerodynamics is one of great interest in the aerospace community. The interest is due to the fact that nonlinear unsteady acrodynamic bchavior can have a significant cffect on the performance and stability of a flight vehicle. It is therefore very important to be able to predict and understand nonlinear unsteady acrodynamic behavior.

Today's most powerful and sophisticated tools for predicting nonlinear unsteady acrodynamics are being developed in the field of computational fluid dynamics (CFD) ${ }^{1}$. The nature and detail of the nonlinear fluid flow that is predicted by a particular flow solver depends on the governing equations that are discretized in the solver. The

*Research Engineer, Member AIAA order of the governing flow equations can vary from the transonic small disturbance (TSD) level to the full NavierStokes equations. As CFD methods improve our ability to predict nonlinear unsteady flows, it is a natural and important step to investigate methods for controlling these flows in order to improve the performance and/or stability of a flight vehicle at flight conditions where nonlinear unsteady aerodynamic effects are significant.

Modern aeroservoelastic (ASE) analysis tools, such as IS AC $^{2}$ (Interaction of Structures, Aerodynamics, and Controls) and ADAM $^{3}$ (Analog and Digital Aeroservoelastic Method), are routinely used for predicting the interaction between the structural system, the aerodynamic system, and the control system of a flexible aircraft so that control laws that account for and take advantage of this flexibility can be designed. The goal of the control law design may be for flutter suppression (stability) ${ }^{4}$ and/or for load alleviation (performance), but, in either case, the control system design has been limited to linear aerodynamic responses. This limitation inhibits the design and analysis of control systems that can account for flow nonlinearities such as shocks, boundary layer effects, and separated flows. Although nonlinear aerodynamics are eventually incorporated into the control system design via wind-tunnel studies or semi-empirical simulations, there is a very real need for modeling nonlinear aerodynamic behavior, such as that predicted by CFD codes, early in the design phase for use in ASE analysis methods. Although some work has been done in directly incorporating simple control laws into CFD $\operatorname{codes}^{5,6}$, these approaches do not generate a general model of the nonlinear aerodynamic response. Instead, the control law gains have to be varied in a trial-and-error manner as flight conditions are varied. Since linear aerodynamic response is modeled as a linear system using rational function approximations in modern ASE codes, the purpose of this research is to investigate the feasibility of modeling nonlinear aerodynamic response as a nonlinear system, in particular, as a Volterra-Wiener nonlinear system.

The Volterra theory was developed by Volterra in $1930^{7}$. The theory is based on functionals, or functions of other functions, and subsequently became a generalization of the linear convolution integral approach that is applied to linear time-invariant (LTI) systems. The theory formulates the response of a nonlinear, timeinvariant system as an infinite sum of multidimensional convolution integrals of increasing order, with the first term in the scries being the standard linear convolution integral. Each multidimensional convolution integral in 
the series has an associated kernel. The first-order kernel is simply the linear unit impulse response of the system and the higher-order kernels are measures of nonlinearity of the system response. This infinite sum of multidimensional convolution integrals is known as the Volterrra Series and it is well defined in both the time and frequency domains.

The Volterra theory has been applied primarily to nonlinear electrical and electronic systems. Wiener 8 contributed significantly to the Volterra theory and, as a result, the theory is currently referred to as the VolterraWiener theory of nonlinear systems. References 9 and 10 developed a kernel identification technique based on autoand cross-correlation functions that can be applied to nonlinear, time-varying systems. The textbooks by Rugh $^{11}$ and Schetzen 12 and the work by Boyd, Chua, and Desoer ${ }^{13}$ and several others ${ }^{14-18}$ are excellent, detailed descriptions of the Volterra-Wiener theory and are highly recommended to the interested reader.

Application of nonlinear system theories, including the Volterra-Wiener theory, to the problem of modeling nonlinear unsteady aerodynamic responses has not been extensive. Ueda and Dowell's ${ }^{19}$ application of the concept of describing functions to unsteady transonic aerodynamic responses is one approach. The work by Tobak and Pearson 20 involved the application of Volterra's concept of functionals to indicial acrodynamic responses for the analytical derivation and experimental determination of nonlinear stability derivatives. The work by Jenkins ${ }^{21}$ is also an investigation into the determination of nonlinear aerodynamic indicial responses and nonlinear stability derivatives. Stalford, Bauman, Garret, and Herdman 22 successfully developed Volterra models for simulating the nonlinear behavior of a simplified nonlinear stall/post-stall aircraft model and the behavior of a simplified model of wing rock.

In Ref. 22, the nonlinear acrodynamic response is analytically defined a priori so that derivation of the Volterra kernels is a straightforward procedure. In general, the nonlinear response of a given configuration at a given flight condition will not be known. The output from a CFD code provides information regarding the nonlinear aerodynamic response of the configuration at a given flight condition to a given input, but a limited amount of information can be inferred regarding the nonlinear acrodynamic response of the configuration to an arbitrary input. Prediction of the nonlinear acrodynamic response of a configuration to an arbitrary input requires identification of the nonlinear kernels of the Volterra Series for the particular configuration.

The problem of Volterra kernel identification has been addressed by Rugh 11 . Clancy and Rugh 23 for discrete systems, Schetzen ${ }^{24}$, and more recently Boyd, Tang, and Chua 25 . There are several ways of identifying the Volterra kernels, both in the time and frequency domains. The methods can be applied to continuous or discrete systems ${ }^{23}$, such as CFD models. Recently, Tromp and Jenkins 26 applied a Laplace domain scheme and aerodynamic indicial responses for the identification of the Volterra kemels of a two-dimensional airfoil undergoing pitching motions using a Navier-Stokes flow solver. The first order, or linear, kernel was identified. The secondorder kernel was identified for a sample problem and the method of Boyd, Tang, and Chua ${ }^{25}$ was suggested for identification of the second-order nonlinear kernel of the airfoil response.

An important characteristic of the Volterra-Wiener theory of nonlinear systems is that a bilinear state-space system can be realized once the nonlinear kernels of interest have been identified 27,28 . This bilinear statespace system can be used as a nonlinear aerodynamic model for acroservoelastic analysis and design. Standard control theory techniques or the theory of bilinear optimal control can then be used for designing control systems that account for nonlinearities in the aerodynamic response.

The objective of the present research is to investigate the application of a time-domain identification technique to the three-dimensional CAP-TSD (Computational Aeroelasticity Program-Transonic Small Disturbance) $\operatorname{code}^{29}$ for identification of the nonlinear, second-order kernel of a NACA64A010 rectangular wing undergoing pitch at transonic Mach numbers. The paper begins with a brief description of the Volterra-Wiener theory of nonlinear systems followed by the description of a kemel identification technique based on unit impulse responses. The kemel identification technique is applied to a simple bilinear state-space system to provide insight into the application of the technique and the nature of a nonlinear kemel. The CAP-TSD code and the computational model of the rectangular wing are then described and, finally, some results for the wing are presented and discussed.

\section{YOLTERRA-WIENER THEORY}

The basic premise of the Volterra-Wiener theory of nonlinear systems is that any nonlinear system can be modeled as an infinite sum of multidimensional convolution integrals of increasing order. This infinite sum is known as the Volterra Series and it has the form

$$
\begin{aligned}
& y(l)=\int_{0}^{l} h_{1}(t-\tau) u(\tau) d \tau+ \\
& \int_{0}^{l} \int_{2 s}^{t} h_{2 s}\left(t-\tau_{1}, t-\tau_{2}\right) u\left(\tau_{1}\right) u\left(\tau_{2}\right) d \tau_{1} d \tau_{2}+\ldots \\
& +\int_{0}^{l} \ldots \int_{0}^{l} h_{n s}\left(t-\tau_{1}, \ldots, l-\tau_{n}\right) u\left(\tau_{1}\right) \ldots u\left(\tau_{n}\right) d \tau_{1} \ldots d \tau_{n}+\ldots
\end{aligned}
$$

where $y(t)$ is the response of the nonlinear system to $u(t)$, an arbitrary input; $h_{1}$ is the first-order kernel or the linear unit impulse response; $h_{2 s}$ is the second-order kernel, and $h_{n s}$ is the $n$th order kernel. It is assumed that:

1). the kernels, input function, and subsequently, the output function are real-valued functions defined for 


$$
\tau_{i} \varepsilon(-\infty,+\infty), i=1, \ldots, n, \ldots
$$

2). the system is causal so that $h_{n s}\left(\tau_{1}, \ldots, \tau_{n}\right)=0$ if any $\tau_{i}<0$

\section{3). the system is time invariant}

Inspection of Equation (1) reveals some very interesting and characteristic features of the Volterra series. If the kemels of order two and above are zero, then the response of the system is linear and is completely described by the unit impulse response $h_{1}(t)$, and the first order convolution integral. The assumption underlying the first order, or linear, convolution integral is that the response of the system at a given timc, $\mathrm{t}$, is independent of the response of the system at a previous time. This is why convolution of a single unit impulse response with an arbitrary input is valid for predicting the response of a linear system. The higher order kernels $h_{n s \text {, are the }}$ responses of the nonlinear systcm to multiple unit impulses, with the number of impulses applied to the system equal to the order of the kernel of interest : c.g., $h_{2 s}$ is the response of the nonlinear system to two unit impulses applied at two varying points in time, $\tau_{1}$ and $\tau_{2}$. The mathematical definition follows directly for the nth order kernels although visualization of these functions can become difficult for orders grcatcr than three. The nonlinear kernels are measures of the relative influence of a previous input on the current response, which is a measure of nonlinearity. This temporal measure of nonlinearity is referred to as memory and, as a result, Volterra systems are sometimes referred to as nonlincar sytems with memory.

The ' $s$ ' in the kernel names stands for 'symmetric' since $h_{2 s}\left(\tau_{1}, \tau_{2}\right)=h_{2 s}\left(\tau_{2}, \tau_{1}\right)$. Although, depending on the domain of integration that is chosen, the kemels can be defined in 'triangular' or 'regular' form, any kernel can be symmetrized without affecting the inpul/output relation. This is done by realizing that

$$
h_{\text {sym }}\left(\tau_{1}, \ldots, \tau_{n)}=(1 / n !) \Sigma h\left(\tau_{\left.\pi(1), \ldots, \tau_{\pi(n)}\right)}\right.\right.
$$

where the indicated summation is over all $n$ ! permutations of the integers 1 through $n$. For the present study, only symmetric kernels will be investigated since these are mathematically easier to interpret and intuitively easier to visualize. For the interested reader, details regarding this issue can be found in Refs. 11 and 12 .

One approach for obtaining Volterra series representations of physical systems is to assume that the system is a 'weakly' nonlinear system. A system that is weakly nonlinear is a system that is well defined by the first few kernels of the Volterra series so that the magnitudes of the kernels greatcr than second or third order fall off rapidly and are negligible. Boyd, Tang, and Chua 25 mention some physical systems that are accuratcly modeled as weakly nonlincar systems including electromechanical and electroacoustic transducers and some biological systems. In this initial study, it is assumed that the nonlinear aerodynamic system that is synthesized from the transonic small-disturbance (TSD) potential cquation is a weakly nonlinear, second-order system. Results are therefore, limited to the identification of the second-order kemel, or $h_{2 s}$.

It should also be noted that the kernels, linear and nonlinear, are input dependent. For example, for a linear system, if the response of the system to an arbitrary input is desired, the unit impulse response of the system due to that particular type of input must first be defined. For a single-input-single-output (SISO) system, there is only one unit impulse response. For a multiple-inputmultiple-output (MIMO) system, there are $\mathrm{nxm}$ unit impulse responses where $n$ is the number of inputs and $m$ is the number of outputs. These unit impulse responses are then combined to form the unit impulse matrix.

\section{Kernel_Identification}

The advantage of the Volterra series approach for modeling nonlinear systems is that once the kernels are identified, the response of the nonlinear system to an arbitrary input can be predicted. The problem of kernel identification, therefore, is central to the successful generation of an accurate Volterra series representation of a nonlinear system. The most obvious approach for identifying the kemels is to derive analytical expressions for the kernels from the governing nonlinear equations of the system of interest ${ }^{30,31}$. Although this approach is applicable to any set of nonlinear equations, including the nonlinear fluid flow equations such as TSD, Euler, and Navier-Stokes equations, it would require some additional coding in order to numerically compute the kernels. Instead, a kernel identification technique is desired that uses the output of a CFD code directly for quick and efficient kernel identification.

Boyd, Tang, and Chua ${ }^{25}$ describe a frequency-domain technique that was successfully applied to the experimental identification of the second-order kemel of a nonlinear electro-acoustic transducer (speaker) system. This and other frequency-domain techniques are available, but it is preferable to use a time-domain kernel identification technique since unsteady, nonlinear CFD analyses are generally performed in the time domain. The time-domain method investigated in this study is the method of unit impulse responses $11,12,24$. Although unit impulse responses are defined for continuous systems, Clancy and Rugh ${ }^{23}$ have shown that an equivalent technique using the unit pulse response can be used for the kernel identification of discrete nonlinear systems.

In what follows, the kernel identification technique using unit impulse responses is derived. The technique is then applied to a simple problem in order to illustrate the discrete application of the technique and the nature of the second-order kernel that is identified. by

A weakly nonlinear, second-order system is described 


$$
\begin{aligned}
& y(t)=\int_{0}^{t} h_{1}(t-\tau) u(\tau) d \tau+ \\
& \int_{00}^{t} \int_{2 s}^{t} h_{2}\left(t-\tau_{1}, t-\tau_{2}\right) u\left(\tau_{1}\right) u\left(\tau_{2}\right) d \tau_{1} d \tau_{2}
\end{aligned}
$$

Inputs consisting of single and double impulse functions can be defined as

$$
\begin{aligned}
& u_{0}(t)=\delta_{0}(t) \\
& u_{1}(t)=\delta_{0}(t)+\delta_{0}\left(t-T_{1}\right)
\end{aligned}
$$

where $T_{1}$ is a distinct positive number. The responses of the system to these two inputs arc

$$
\begin{aligned}
y_{0}(t)= & h_{1}(t)+h_{2 s}(t, t) \\
y_{1}(t)= & h_{1}(t)+h_{1}\left(t-T_{1}\right)+h_{2 s}(t, t)+ \\
& 2 h_{2 s}\left(t, t-T_{1}\right)+h_{2 s}\left(t-T_{1}, t-T_{1}\right)
\end{aligned}
$$

The $2 h_{2 s}\left(t, t-T_{1}\right)$ term is a result of the symmetry of the kernel since $h_{2 s}\left(t, t-T_{1}\right)=h_{2 s}\left(t-T_{1}, l\right)$. Then

$$
\begin{aligned}
& y_{1}(t)= y_{0}(t)+h_{1}\left(t-T_{1}\right)+2 h_{2 s}\left(t, t-T_{1}\right)+ \\
& h_{2 s}\left(t-T_{1}, t-T_{1}\right)
\end{aligned}
$$

and noticing that

$$
y_{0}\left(t-T_{1}\right)=h_{1}\left(t-T_{1}\right)+h_{2 s}\left(t-T_{1}, t-T_{1}\right)
$$

results in

$$
y_{1}(t)=y_{0}(t)+y_{0}\left(t-T_{1}\right)+2 h_{2 s}\left(t, t-T_{1}\right)
$$

Solving for the second-order kernel

$$
h_{2 s}\left(t, t-T_{1}\right)=(1 / 2)\left(y_{1}(t)-y_{0}(t)-y_{0}\left(t-T_{1}\right)\right)
$$

which is the value of the second-order kcrnel for any value of $\mathrm{T}_{1}$.

The procedure for computing $h_{2 s}$ is as follows. First, $\mathrm{y}_{0}(\mathrm{l})$, which is the response of the system to a unit impulse response applied at time $l$, is generated. Then, since the system is time invariant, yo is shifted in time to a new time $t-T_{1}$, which becomes $y_{0}\left(t-T_{1}\right)$. The response of the system to two unit impulses, one at time $t$ and one at time $t-T_{1}$, or $y_{1}(t)$, is gencrated and finally, all three responses are substituted into equation (4). The second-order kernel, $h_{2 s}$ is a two-dimensional function of time. That is, it is a function of time $l$ and a function of time lag $T_{1}$ so that for every value of $T_{1}$ that is used, a new function of time $t$ is defined. These sets of functions of time are referred to as "terms" of the kernel. The first term of $h_{2 s}$ is defined when $T_{1}=0$, or when both unit impulse inputs are applied at the same point in time. When $T_{1}=0$, cquation (4) reduces to

$$
\begin{aligned}
h_{2 s}(t, t) & =(1 / 2)\left(y_{1}(t)-y_{0}(t)-y_{0}(t)\right) \\
& =(1 / 2) y_{1}(t)-y_{0}(t)
\end{aligned}
$$

The second term of the kernel depends on the next value of $T_{1}$ selected. The number of $T_{1}$ 's, or the number of terms, needed to accurately define a second-order kemel depends on the nonlinear system under investigation.

In addition, the linear portion of the nonlinear response can be identified when $T_{1}=0$. It is important to realize that the linear portion of the nonlinear response is not, in general, equivalent to the purely linear response. For example, for an aerodynamic system, the linear response computed using the linear equations (flat plate) is not identical to the linear portion of the response computed using the nonlinear equations (thickness).

The linear portion of the nonlinear response is defined as follows. The response of the system represented by equation (3) to $2 \mathrm{u}_{0}(\mathrm{t})$ is

$$
y_{2}(t)=2 h_{1}(t)+4 h_{2 s}(t, t)
$$

Then, solving simultancously with $\mathrm{y}_{0}(\mathrm{t})$ results in

$$
h_{1}(t)=2 y_{0}(t)-(1 / 2) y_{2}(t)
$$

which is the unit impulse response of the linear portion of the nonlinear response.

The equations derived above for $h_{2 s}$ are clear and simple measures of nonlinearity. In these equations, nonlinearity is measured as a deviation from linear bchavior. This is evident in that $h_{2 s}$ is identically zero for a linear system due to the principle of linear superposition. Therefore, an additional benefit of the second-order kemel is that it can be used to measure the true linearity of a system that is classified as being linear or for establishing boundaries beyond which the assumptions of linearity begin to fail. Definitions of higher order kernels can be derived in the same way as for $h_{2 s}$ by applying the appropriate number of unit impulses to the system.

Once $\mathrm{h}_{2 \mathrm{~s}}$ is identified, the nonlinear response of the weakly nonlinear, second-order system to an arbitrary input can be determined. A further advantage of the Volterra theory of nonlinear systems is that a bilinear state-space system can be realized once the kernels are identified, generating a nonlinear aerodynamic state-space system that is amenable for use with modern control theory. The relationship between the Volterra kemels and the bilinear state-space formulation is as follows.

It is well known that, for a linear system described by the following state-space representation

$$
\begin{aligned}
& \dot{x}=A x+B u \\
& y=C x
\end{aligned}
$$

(where the D matrix, or feedthrough term, has been set to zero) the system's unit impulse response is given by 


$$
h(t)=C(\exp (A t)) B
$$

If the unit impulse response of a lincar system is known, then a state-space realization can bc obtained using standard linear system realization techniques. In a natural extension to this concept, a Voltcrra system can be modeled by a bilinear state-space systcm as follows

$$
\begin{aligned}
& \dot{x}=A x+N x u+B u \\
& y=C x
\end{aligned}
$$

where the matrix $\mathbf{N}$ is a measure of the nonlincarity of the system. The system is linear when $\mathrm{N}=0$, as it is reduced to equation (7). The relationship between the second-order Volterra kemel and the bilinear state-space formulation is

$$
h_{2 \operatorname{reg}}\left(t_{1}, t_{2}\right)=C\left(\exp \left(A t_{1}\right)\right) N\left(\exp \left(A t_{2}\right)\right) B
$$

where the subscript 'reg' stands for the 'regular' definition of the kernel. Equation (2) can then be used to compute the symmetric kernel $h_{2 s}$ from $h_{2 \mathrm{reg}}$.

\section{EXAMPLE PROBLEM}

Assume a system described by the following differential equation

$\ddot{y}(t)+a_{1} \dot{y}(t)+a_{0} y(t)=b_{0} u(t)+n_{0} y(t) u(t)$

In bilinear state-space form, equation (11) can be rewritten as equation (9) where

$$
A=\left[\begin{array}{cc}
0 & 1 \\
-a_{0} & -a_{1}
\end{array}\right], N=\left[\begin{array}{ll}
0 & 0 \\
n_{0} & 0
\end{array}\right], B=\left[\begin{array}{l}
0 \\
b_{0}
\end{array}\right],
$$

and $C=\left[\begin{array}{ll}1 & 0\end{array}\right]$

Values for the $A, N$, and $B$ matrices werc arbitrarily chosen as $a_{0}=2$., $a_{1}=3 ., n_{0}=-6$., and $b_{0}=1$. The linear response of this system is obtained by selting $n_{0}=0$. An arbitrary input, shown in the inset of figurc 1 , was defined as

$$
\begin{aligned}
u_{\text {arb }}(t) & =0.0, t<.1 \text { and } t>1.1 \\
& =t-.1, .1 \leq t \leq 1.1
\end{aligned}
$$

and applied to discretized versions of the linear $(\mathrm{N}=0)$ and nonlinear (bilinear) systems in order to examine the differences in the responses due to this arbitrary input using a time step of 0.01 . The responses can be seen in figure 1 where it is clear that the effect of the added nonlinearity is to reduce the level of the linear response.

Application of the kernel identification technique to continuous systems requires the usc of unit impulse inputs. For discrete systems, the equivalent of a unit impulse input is a unit pulse input defined as

$$
\begin{aligned}
u_{p}(t) & =1,, t=t 0 \\
& =0, \quad t \neq t 0
\end{aligned}
$$

where $t 0$ can be any time step since the system is time invariant. Also, the unit impulse response of a continuous system is approximately equal to the unit pulse response of the discretized version of the same system divided by the time step used in the discretization. The discrete responses are therefore divided by the time step so that comparisons with the continuous, or analytical, responses can be made.

The unit pulse input, $u_{p}(t)$, was applied to the discretized linear system and the resultant unit pulse response was compared to the analytical (or continuous) unit impulse response of the system from equation (8). This comparison is presented in figure 2 , showing excellent agreement. The slight difference between the two responses is a result of the time step (DT $=0.01$ ) that was used. A smaller time step improves the accuracy of the unit pulse response.

The unit pulse input was then modified to include two unit pulses for identification of the second-order kemel so that

$$
\begin{aligned}
u_{p}(t) & =1 ., t=t 0 \text { and } t=t 1 \\
& =0, \quad t \neq t 0 \text { and } t \neq t 1
\end{aligned}
$$

where the value of $t 1$ is a time step such that $t 1-t 0=T_{1}$. The to was held fixed while the $t 1$ was varied in order to vary $T_{1}$. The value of $T_{1}$ was varied in increments of ten time steps, or 0.1 seconds. The first term of the secondorder kernel corresponds to $T_{1}=0.0$; the second term of the second-order kernel corresponds to $T_{1}=10$ time steps ; the third term corresponds to $T_{1}=20$ time steps and so on. A total of twenty terms were generated and the resulting second-order kernel is shown in figure 3 as a function of time $t$ and time lag $T_{1}$. In figure $3, T B$ is the second term of the kernel $\left(T_{1}=10\right.$ time steps), $T C$ is the third term, TD is. the fourth term, and the first term, TA, is not visible because it was zero. As can be seen, the secondorder kernel is a two-dimensional function that varies smoothly with time $t$ and time lag $T_{1}$. It can also be seen that the second-order nonlinear response of the system at each time lag $T_{1}$ exhibits a rapid initial growth, reaches a maximum response and then begins to dissipate. The fact that the second-order kernel is negative is consistent with the result in figure 1 where it was shown that the added nonlinear terms reduced the response of the system from the purely linear response. Inspection of a nonlinear kernel, therefore, can provide a significant amount of information regarding the behavior of a nonlinear system in terms of amplitude and time lag variations. This is important information for the design of effective control systems.

The exact, analytical second-order kernel of the bilinear system was computed using equation (10). The analytical second-order kemel is not a symmetric kemel and so it must be symmetrized before it can be compared with the numerically-identified symmetric second-order 
kerncl. Symmetrization of the analytical kernel is performed by using equation (2). The numericallyidentified, or discrete, kernel is divided by the square of the time step so that it can be compared with the analytical, or continuous, kernel. The symmetrized analytical second-order kernel and the symmetric, numericallyidentified second-order kemel are shown in figure 4 for two time lags, TD (30 time steps) and TG (60 time steps). The comparison is excellent with slight differences occuring around the regions of maximum response. Again, improved accuracy can be achieved by using a smaller time step in the numcrical identification technique. The important point to be made here, however, is that the use of discrete, unit pulses can be used to identify the discrete, second-order Volterra kernel of a discrete nonlinear system.

\section{COMPUTATIONAL_PROCEDURES}

The CAP-TSD program is a finite-difference program which solves the general-frequency modified transonic small-disturbance (TSD) potential equation. The TSD potential equation is defined by

$$
\begin{aligned}
M_{\infty}^{2}\left(\phi_{t}+2 \phi_{x}\right)_{t}= & {\left[\left(1-M_{\infty}^{2}\right) \phi_{x}+F \phi_{x}^{2}+G \phi_{y}^{2}\right]_{x}+} \\
& \left(\phi_{y}+H \phi_{x} \phi_{y}\right)_{y}+\left(\phi_{z}\right)_{z}
\end{aligned}
$$

where $M_{\infty}$ is the freestream Mach number, $\phi$ is the disturbance velocity potential, and the subscripts of $\phi$ represent partial derivatives.

Several choices are available for the coefficients F, G, and $\mathrm{H}$ depending upon the assumplions used in deriving the TSD equation. For transonic applications, the coefficients are herein defined as

$$
\begin{aligned}
& F=-\frac{1}{2}(\gamma+1) M_{\infty}^{2} \\
& G=\frac{1}{2}(\gamma-3) M_{\infty}^{2} \\
& H=-(\gamma-1) M_{\infty}^{2}
\end{aligned}
$$

where $\gamma$ is the ratio of specific heals. The linear potential equation is recovered by simply setting $F, G$, and $\mathrm{H}$ cqual to zero.

Equation (12) is solved within CAP-TSD by a timeaccuratc approximate factorization (AF) algorithm developed by Batina 29 . In Refs. 32 and 33, the AF algorithm was shown to be efficient lor application to stcady or unsteady transonic flow problems. Several algorithm modifications have been made which improve the stability of the AF algorithm and the accuracy of the results ${ }^{34}$. One of these improvements is the option to include vorticity and entropy corrections 35 for improved shock modeling. This option, however, was not used for the analyses presented in this paper.
The CAP-TSD program can treat configurations with combinations of lifting surfaces and bodies including canard, wing, tail, control surfaces, tip launchers, pylons, fuselage, stores, and nacelles. The code was recently applied to the Active Flexible Wing (AFW) wind-tunnel model, which included modeling of the fuselage and tip stores, for prediction of the model's transonic aeroelastic behavior ${ }^{36}$.

The code has an exponential pulse capability that can be used for generating unsteady acrodynamic pitch, plunge, and modal responses. The pulse and pulse rate are defined as

$$
\begin{aligned}
& p(t)=\delta_{0} \exp \left(-w(t-t c)^{2}\right) \\
& \dot{p}(t)=-2 w(t-t c) p(t)
\end{aligned}
$$

where $t$ and $t c$ are in terms of nondimensional time steps. For pitching motions, the angle-of-attack input function is defined using equation (14) and the rate of change of angle of attack, or angle-of-attack rate is defined using equation (15). These functions of time then become part of the downwash equation which, for simple pitching motions is

$$
\mathrm{f}(\mathrm{x}, \mathrm{l})^{ \pm}=\frac{\mathrm{d} t^{ \pm}}{\mathrm{dx}}-\alpha(\mathrm{l})-\dot{\alpha}(\mathrm{t})\left(\mathrm{x}-\mathrm{x}_{\text {pitch }}\right) / \mathrm{U}_{\infty}
$$

where the plus and minus signs indicate upper and lower surfaces of the airfoil. The first term in equation (16) is the airfoil geometry slopes, followed by the angle of attack, and by the angle-of-attack rate multiplied by the pitch distance where $x_{\text {pitch }}$ is the pitch axis. The downwash provides the boundary condition defined at the $\mathrm{z}=0$ plane required to complete the solution of the TSD equations. For the linear aerodynamic solution, or flat plate solution, the airfoil geometry slopes are zero and the downwash becomes a function of angle of attack and angle-of-attack rate only.

The computational grid of the NACA64A010 rectangular wing is dimensioned 137 by 40 by 84 grid points in the $x-, y-$, and $z$-directions respectively. The wing has an aspect ratio of three but the computational domain covers only one semi-span due to flow symmetry.

\section{RESULTS AND DISCUSSION}

Before the kernel identification technique was applied to an acrodynamic system, the appropriate excitation input had to be defined. It was obvious that the excitation input had to be a perturbation of the downwash, equation (16). The excitation input could, therefore, be composed of onc or a combination of the parameters that define the downwash. In addition, the excitation input had to be of an "impulsive" nature so that the theoretical assumptions used in the derivation of the kernel identification technique were maintained.

The requirement that the excitation input be "impulsive" disallowed the use of the exponential pulse capability defined in the CAP-TSD code, eqs. (14) and (15). The acrodynamic response induced by an exponential pulse input is a response of the aerodynamic 
system to a smoothly-varying function of angle of attack and a smoothly-varying function of angle-of-atlack rate. This response cannot be referred to as the unit pulse response of the system and therefore cannot be used in the linear convolution integral to predict the linear response of the system or in the identification of the nonlinear kernels. The correct excitation input should, therefore, be of a unit magnitude and should be applied at only one time step, as was presented for the example problem earlier.

Based on this reasoning, the unit pulse inputs available are then

$$
\begin{aligned}
& \alpha(t)=0.01745 \operatorname{rads}(1 \mathrm{deg}), t=t 0 \\
& =0.0, t \neq 10 \\
& \text { with } \dot{\alpha}(t)=0.0 \text { everywhere } \\
& \dot{\alpha}(\mathrm{t})=1 \mathrm{rad} / \mathrm{sec}, \mathrm{t}=10 \\
& =0.0, t \neq 10
\end{aligned}
$$

or a combination of both anglc of attack and angle-ofattack rate inputs. Also, due to the impulsive nature of the unit pulse inputs, a very small time step of DT $=0.0001$ had to be used in order to obtain smooth acrodynamic responses. All dynamic responses, linear and nonlinear, were 500 time steps in length. It should be noted that this choice of time step and number of time steps results in only .05 chords of travel. This very small time sample is dominated by the high frequency content of the response so that the results that follow are limited to high frequency responses.

The application of the unit anglc-of-allack pulse input resulted in very small aerodynamic responses for both the linear (flat plate) and the nonlinear (thickness) aerodynamic solutions using CAP-TSD. An attempt to identify the nonlinear kerncl using thesc responses resulted in numerical noise and was not possible. The second type of input, the unit angle-of-attack rate pulse input, provided sufficient excitation of the nonlinear equations so that a nonlinear kernel could be identilied, as will be seen. The combined unit anglc-of-allack and unit angle-of-attack rate inputs resulted in responses that were only marginally different from the unit angle-of-attack ratc responses. As a result of these preliminary investigations, all subsequent analyses are based on unit angle-of-attack rate pulse inputs only. Also, only lift coefficient responses to pitching motions about the quarter-chord location are investigated in this study, although the techniques presented are applicable to any force coefficient response including gencralized acrodynamic forces.

For the results that follow, $\mathrm{P} 1$ is the unit pulse response at time $10 ; \mathrm{P} 2$ is the unit pulse response at time $t=t 0+T_{1} ; P 12$ is the response duc to a unit pulse at time $\mathrm{t}=\mathrm{t} 0$ and another unit pulse at time $\mathrm{t}=\mathrm{t} 0+\mathrm{T}_{1}$; and $\mathrm{P} 11$ is the response due to two unit pulses at time $t=t 0$. The unit angle-of-attack rate inputs were applied at the 60th, 110 th, and 160th time steps which translates to time lags equal to $0.0,50.0$, and 100.0 time steps. The choice of these time steps was arbitrary and the choice of the 60th time step as the first response, or as the $\mathrm{P} 1$ response, was done in order to avoid any numerical transients that might occur when the nonlinear aerodynamic analyses are initiated from stcady-state solutions.

\section{Linear KerneL_Identification}

The P1 linear (flat plate) lift-coefficient response shown in figure 5 has the characteristics that are typical of a unit pulse response. That is, the initial part of the response is impulsive and the latter part of the response is a damped transient. It should be restated that the time step at which the input is applied is not important since the linear system is time invariant.

The linear convolution integral,

$$
y(t)=\int_{0}^{t} h(t-\tau) u(\tau) d \tau
$$

was used to verify that P1 (figure 5) was indeed a unit pulse response. This was done by first generating a linear (llat plate) lift-coefficient response to an arbitrary input using the CAP-TSD exponential pulse capability, eqs. (14) and (15) at $M=0.85$. The pulse was defined with a $w=90,000 ., \delta_{0}=0.009$ rads $(.5 \mathrm{deg})$, and centered at tc $=250$ time steps. The response to this exponential pulsc, which will be refered to as the linear arbitrary response, is shown in figure 6 along with the angle of attack (inset) and the corresponding angle-of-attack rate functions generated by eqs. (14) and (15). It should be noted that this response is of a relatively high-frequency which, as can be seen in figure 6 , is influenced primarily by the angle-of-attack rate input.

The linear convolution integral was then evaluated using the angle-of-attack rate function, presented in figure 6 , as the input, $u$, and the $P 1$ response shown in figure 5 as the unit impulse response, $h$. A comparison of the linear arbitrary response shown in figure 6 and the response computed from the linear convolution of $P 1$ and the angle-of-attack rate function is presented in figure 7. The excellent agreement between the two responses verifies the use of the unit angle-of-attack rate pulse input for generating unit pulse responses that can be used for predicting high-frequency arbitrary responses. However, accuratc prediction of low-frequency responses, where the angle of attack input becomes significant, needs further investigation.

\section{Nonlinear Kernel_Identification}

The nonlinear aerodynamic responses were computed about steady-state converged solutions of the NACA64A010 rectangular wing at zero degrees angle of altack. These steady-state solutions consisted of about 2500-5000 time steps using a time step DT=0.01. The gas medium used in the nonlinear analyses was air which 
corresponds to a $\gamma=1.4$. The assumption of time invariance was verified for the nonlinear aerodynamic responses.

At $M=0.85$, in the steady solution, a strong shock is present at the $60 \%$ chord location so that a level of nonlinear response should be noticed when identification of the second-order kernel is performed. The nonlinear P1, P2, P12 and P11 responses of the NACA64A010 wing at $M=0.85$ are shown in figure 8 . The linear portion of the nonlinear response, or $h_{1}$ (eq. (6)), is computed first as

$$
h_{1}=2(\mathrm{P} 11)-.5(\mathrm{P} 1)
$$

The linear unit pulse response from the linear (flat plate) solution is ' $h$ ' and should not be confused with ' $h{ }_{1}$ '. Figure 9 is a comparison of these two unit pulse responses where the difference is small but noticeable, in terms of an associated time lag for the $h_{1}$.

The first term of the second-order kernel was computed using

$$
\mathrm{H} 2 \mathrm{~S} 1=.5(\mathrm{P} 11-\mathrm{P} 1-\mathrm{P} 1)=.5(\mathrm{P} 11)-\mathrm{P} 1
$$

which corresponds to a time lag $\mathrm{T} 1=0.0$. The nonlinear $\mathrm{P} 2 \mathrm{~A}$ and $\mathrm{P} 12 \mathrm{~A}$ responses were computed using a time lag $T_{1}=50.0$ and the nonlinear $P 2 B$ and $P 12 B$ responses were computed using a time lag $T_{1}=100.0$. The $P 2 A, P 12 A$, $\mathrm{P} 2 \mathrm{~B}$, and $\mathrm{P} 12 \mathrm{~B}$ responses were used for computing the second and third terms of the sccond-order kernel, respectively, as

$$
\begin{aligned}
& \mathrm{H} 2 \mathrm{~S} 2=.5(\mathrm{P} 12 \mathrm{~A}-\mathrm{P} 2 \mathrm{~A}-\mathrm{P} 1) \\
& \mathrm{H} 2 \mathrm{~S} 3=.5(\mathrm{P} 12 \mathrm{~B}-\mathrm{P} 2 \mathrm{~B}-\mathrm{P} 1)
\end{aligned}
$$

Notice that computation of additional terms of the secondorder kernel requires only the generation of the appropriate $P 12$ response since the $P 1$ response needs to be computed only once and the $P 2$ response is just the $\mathrm{Pl}$ response shifted in time.

The three terms of the $\mathrm{M}=0.85$ second-order kernel, $\mathrm{H} 2 \mathrm{~S} 1, \mathrm{H} 2 \mathrm{~S} 2$, and $\mathrm{H} 2 \mathrm{~S} 3$, are shown in figure 10 . It can be seen that the identified second-order kerncl, although noisy at the smaller magnitudes, does exhibit a particular shape which is not numerical noisc. The small values of the $\mathrm{M}=0.85$ second-order kernel are indications that the nonlincarities at this condition are small. This is also consistent with the result presented for the example problem where the second-order kernel identified for that case was on the order of 1.e-06. Also, the approach to zero values of the second-order kernel as time lag is increased, or as more terms are computed, is as expected since the second-order kernel is a finite and bounded function of time. The $M=0.85$ second-order kernel therefore exhibits behavior that is characteristic of a second-order kemel.

The same three terms of the second-order kernels at $M=0.5$ and $M=0.93$ were also identified. At $M=0.93$, the shock was located at the trailing edge. Figure 11 is a comparison of the nonlinear unit pulse responses of the
NACA64A010 rectangular wing at $M=0.5,0.85$, and 0.93 . It is noticed that the amplitude and frequency of these unit pulse responses decreases as Mach number is increased. This, of course, is configuration and motion dependent so that for a plunging motion, for example, this same trend may not occur. Comparison of the second-order kernels for all three Mach numbers reveals a suprising and interesting result. Figure 12 presents the three terms of the $M=0.5$ second-order kemel and figure 13 presents the three terms of the $M=0.93$ second-order kernel. At first glance, and comparing with the three terms of the $M=0.85$ second-order kernel (figure 10), it appears that the magnitude of the second-order kernels decreases with increasing Mach number. This is the reverse trend that is expected since the second-order kernel should increase with increasing levels of nonlinearity, or, in this case, with an increase in Mach number.

This preliminary comparison is not complete. An appropriate comparison of the second-order kemels of the three Mach numbers should account for the differences in the magnitude of the unit pulse responses at each Mach number. If, for example, the maximum absolute value of each of the three terms of the second-order kernels is divided by the maximum value of the unit pulse response of each corresponding Mach number, the resulting values would be better indicators of the relative magnitude of the nonlinear effects. A bar chart of these values, referred to as the relative nonlinearity, for the three Mach numbers is presented in figure 14 for all three terms of the secondorder kernels. Figure 14 does indeed show the growth of relative nonlinearity as Mach number is increased for all three terms of the second-order kernels and, in particular, the sudden increase in the first term at $\mathbf{M}=0.93$.

A qualitative interpretation of the second-order kernels identified at $M=0.5, M=0.85$, and $M=0.93$ would imply that a nonlinear response at these Mach numbers is dominated by an in-phase increase in magnitude (the first tcrm) and subsequent, but smaller, time-lagged variations in the response. This is, in fact, a reasonable interpretation as can be seen in figure 15 , which is a comparison of the linear (flat plate) aerodynamic and nonlinear (thickness) acrodynamic responses at $\mathrm{M}=0.85$ due to the same exponential pulse function described in figure 6. The nonlinear response is larger in magnitude at the regions of maximum response and exhibits some phase difference with respect to the linear response.

The results presented thus far are encouraging and support the feasibility and applicability of the Volterra series approach for the modeling of nonlinear unsteady acrodynamic responses. Additional work is needed for determining the minimum number of terms of the secondorder kernel that are required for accurate prediction of responses to arbitrary inputs, in applying the unit pulse response technique to lower frequency responses where the effect of angle of attack inputs will be more significant, and in evaluating the third order kerncls for verification of the assumption of a weakly nonlinear system.

\section{CONCLUSIONS}

The Volterra-Wiener theory of nonlinear systems was bricfly described and presented as a method for modeling 
nonlinear aerodynamic responses for use in aeroservoelastic (ASE) analysis and design. Successful application of the theory is contingent upon the sucessful identification of the nonlinear kernels. Although several methods for identifying the kernels exist, the method investigated in this study was a time-domain technique, based on unit impulse responses. The technique was described and applied to a simple bilinear (nonlinear) system for illustrative purposes and to verify the application of the technique to discrete nonlinear systems. The first order, or linear, kerncl and the second-order nonlinear kernel of the simple system were accurately identified using the technique. Interpretations of the second-order kemel were also presented.

The kernel identification technique was then applied to a NACA64A010 rectangular wing using the CAP-TSD code. Application of the kernel identification technique to the aerodynamic model began with the identification of the first order kernel due to the lincar acrodynamic response (flat plate solution) of the wing in pitch about the quarter-chord location. It was shown that a unit impulse response, or, for discretc systems, a unit pulse response, can be accurately computcd by a unit cxcitation of rate of angle of attack in the downwash function for predicting linear high-frequency responses. Additional work needs to be performed for prediction of low frequency responses.

Identification of the second-order kernel due to pitching motions about the quarter-chord location was then performed using the same unit angle-of-atlack rate pulse input that was used for identification of the linear kernel. Nonlinear (transonic) acrodynamic unit pulse responses were computed at $M=0.5,0.85$, and 0.93 where strong shocks are present at the $\mathrm{M}=0.85$ and the $\mathrm{M}=0.93$ conditions. Three terms of the second-order kernels for the three Mach numbers were then identified and discussed. The results are encouraging, although additional effort is required for determining the number of terms of the second-order kernel that are required for accurate prediction of nonlinear responses to arbitrary inputs and in verifying that the assumption of a weakly nonlinear second-order system is accurate for transonic aerodynamic responses.

\section{REEERENCES}

${ }^{1}$ Edwards, J. W.; and Thomas, J. L. : Computational Methods for Unsteady Transonic Flows, Chapter 5 in Unsteady Transonic Aerodynamics, Editcd by David Nixon, Progress in Astronautics and Acronautics, Volume $120,1989$.

2Peele,Elwood L.; and Adams, William M., Jr. : "A Digital Program for Calculating the Interaction Betwecn Flexible Structures, Unsteady Acrodynamics, and Active Controls," NASA TM-800040, January 1979.

${ }^{3}$ Noll, T. E. ; Blair, M. ; and Cerra, J. : "An Aeroservoelastic Analysis Method for Analog or Digital Systems," Journal of Aircraft, November 1986.
${ }^{4}$ Perry, B. III; Mukhopadhyay, V.; Hoadley, S. T.; Cole, S. R.; Buttrill, C. S.; and Houck, J. A. : "Design, Implementation, Simulation, and Testing of Digital Flutter Suppression Systems for the Active Flexible Wing Wind-Tunnel Model," ICAS Paper Number 90. 1.3.2, Presented at the 17th International Council of the Aeronautical Sciences, Stockholm, Sweden, September 914, 1990.

5Ide, H. ; and Ominsky, D. : "Simulation of Static and Dynamic Acroclastic Behavior of a Flexible Wing with Multiple Control Surfaces," AIAA Paper Number 901075, Presented at the 31st Structures, Structural Dynamics, and Materials Conference, Long Beach, CA, April 2-4,1990.

$6_{\text {Lee, Elizabeth M.; and Batina, J. T. : "Conical Euler }}$ Simulation and Active Suppression of Delta Wing Rocking Motion," NASA TM-102683, October 1990.

${ }^{7}$ Volterra, V.: Theory of Functionals and of Integral and Intcgro-Differential Equations. Dover Publications, Inc., New York, 1959.

${ }^{8}$ Wiener, N.: "Response of a Nonlinear Device to Noise," Report No. 129, Radiation Laboratory, M.I.T., Cambridgc; Massachusetts, April 1942. (Also published as U.S. Department of Commerce Publications PB58087.)

${ }^{9}$ Lee, Y. W.: and Schetzen, M.: “Measurement of the Wiener Kernels of a Non-linear System by Crosscorrelation," International Journal of Control. Volume 2(3), pp. 237-254, September 1965.

$10 \mathrm{Ku}$, Y. H.; and Su, C. C.: "Volterra Functional Analysis of Nonlinear Time-Varying Systems," Journal of the Franklin Institute, Volume 204, Number 6, December 1967.

${ }^{1}$ Rugh, Wilson J. : Nonlinear System Theory. The Volterra-Wiener Approach The Johns Hopkins University Press, 1981.

${ }^{12}$ Schetzen, Martin : The Volterra and Wiener Theories of Nonlincar Syslems, John Wilcy \& Sons, 1980.

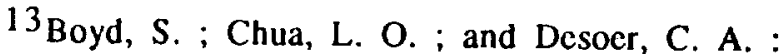
"Analytical Foundations of Volterra Series," IMA Journal of Mathematical Control \& Information, 1984, Volume 1, pp. 243-282.

${ }^{14}$ Brilliant, M. B.: "Theory of the Analysis of Nonlinear Systems," Technical Report 345, M.I.T. Rescarch Laboratory of Electronics, March 1958.

15 Boyd,S.P.: "Volterra Series: Engineering Fundamentals," PhD Dissertation, University of California, Berkeley, 1985. 
16 Boyd, S. P.; and Chua, L. O.: "Fading Memory and the Problem of Approximating Nonlincar Operators with Volterra Series," IEEE Transactions on Circuits and Systems, Volum CAS-32, Number 11, November 1985.

${ }^{17} \mathrm{Ku}$, Y. H.; and Wolf, A. A.: "Volterra-Wiener Functionals for the Analysis of Nonlinear Systems," Journal of the Franklin Institute, Volume 281, Number 1, January 1966.

18 Bedrosian, E.; and Rice, S. O.: "The Output Properties of Volterra Systems (Nonlinear Systems with Memory) Driven by Harmonic and Gaussian Inputs," Proceedings of the IEEE, Volume 59. Number 12, December 1971.

${ }^{19}$ Ueda, T.; and Dowell, E.H.: "Flutter Analysis Using Nonlinear Aerodynamic Forces," Presented at the 23rd AIAA/ASME/ASCE/AHS Structures, Structural Dynamics, and Materials Conferencc, New Orleans, LA, May $10-12,1982$.

${ }^{20}$ Tobak, Murray ; and Pearson, Walter E. : "A Study of Nonlinear Longitudinal Dynamic Stability," NASA Technical Report R-209, September 1964.

21 Jenkins, J. E.: "Relationships Among Nonlinear Aerodynamic Indicial Response Models, Oscillatory Motion Data, and Stability Derivatives," Paper No. CP89-3351, Presented at the AIAA Atmospheric Flight Mechanics Conference, Boston, Massachusclts, August 14-16, 1989.

${ }^{22}$ Stalford, H.; Baumann, W. T. ; Garrell, F. E. ; and Herdman, T. L. : "Accurate Modeling of Nonlinear Systems Using Volterra Series Submodels," Presented at the 1987 American Control Conference, Minncapolis, MN, June 10-12, 1987.

${ }^{23}$ Clancy, S. J.; and Rugh, W. J.: "A Note on the Identification of Discrete-Time Polynomial Systems," IEEE Transactions on Automatic Control, Volume AC. 24, Number 6, December 1979.

${ }^{24}$ Schetzen, M.: "Measurement of the Kernels of a Nonlinear System of Finite Order,"International Journal of Control, Volume 1(3), pp.251-263, March 1965; Corrigendum, Vol. 2(4), p.408, Octobcr, 1965.

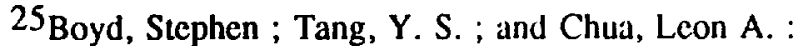
"Measuring Volterra Kemels," IEEE Transactions on Circuits and Systems, Volume CAS-30, Number 8, August 1983.

${ }^{26}$ Tromp, J. C.; and Jenkins, J. E.: "A Volterra Kernel Identification Scheme Applied to Acrodynamic Reactions," AIAA Paper No. 90-2803, Presented at the AIAA Atmospheric Flight Mechanics Conference, Portland, Oregon, August 20-22, 1990.
${ }^{27}$ Clancy, S. J.; and Rugh, W. J.: "On the Realization Problem for Stationary, Homogenous, Discrete-Time Systems," Automatica, Volume 14, 1978, pp. 357-366.

${ }^{28}$ King, A. M.; and Skelton, R. E.: "Model Reduction for Discrete Bilinear Systems," IEEE Transactions on Automatic Control, Volume AC-24, Number 6, December 1979.

${ }^{29}$ Batina, J. T. : "Efficient Algorithm for Solution of the Unsteady Transonic Small-Disturbance Equation," Journal of Aircraft, Volume 25, July 1988, pp. 598-605.

${ }^{30}$ Flake, R. H. : "Volterra Series Representation of Nonlinear Systems," Transactions of the American Institute of Electrical Engineers, Volume 81, 1962, Part II:Applications and Industry.

31 Ibrahim, R. A. : "Response Analysis of Nonlinear Systems Using Functional-Perturbational Type Approach," 6th International Modal Analysis Conference, February 1-4, 1988, Kissimmee, FL.

32 Batina, J. T.; Seidel, D. A.; Bland, S. R.; and Bennett, R. M. : "Unsteady Transonic Flow Calculations for Realistic Aircraft Configurations," Journal of Aircraft, Volume 26, January 1989, pp. 21-28.

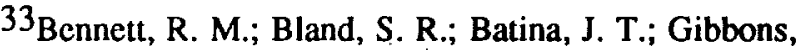
M. D.; and Mabey, D. G. : "Calculation of Steady and Unsteady Pressures on Wings at Supersonic Speeds with a Transonic Small-Disturbance Code," AIAA Paper Number 87-0851, Presented at the AIAA/ASME/ASCE/AHS 28th Structures, Structural Dynamics, and Materials Conference, Monterey, CA, April 6-8, 1987.

${ }^{34}$ Batina, J. T.: "Unsteady Transonic Algorithm Improvements for Realistic Aircraft Applications," Journal of Aircraft, Volume 26, February 1989, pp. 131. 139.

35 Batina, J. T.: "Unsteady Transonic Small-Disturbance Theory Including Entropy and Vorticity Effects," Journal of Aircraft, Volume 26, Number 6, June 1989, pp. 531 538.

36 Silva, W. A.; and Bennett, R. M.: "Predicting the Aeroelastic Behavior of a Wind-Tunnel Model Using Transonic Small Disturbance Theory," Presented at the 17th Congress of the International Council of the Acronautical Sciences, Stockholm, Sweden, September 914, 1990. 


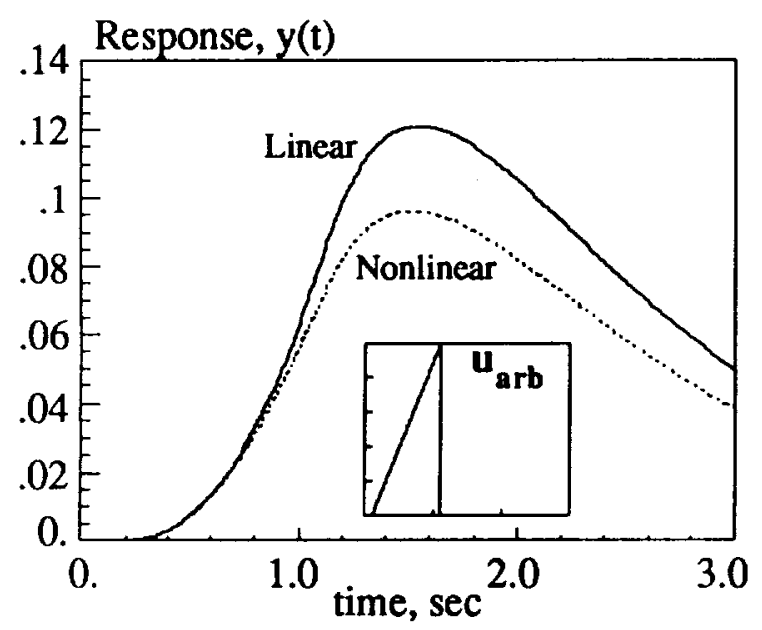

Figure 1 The response of the linear system $(\mathrm{N}=0)$ and the nonlinear (bilinear) system due to the input shown in the inset for the example problem.

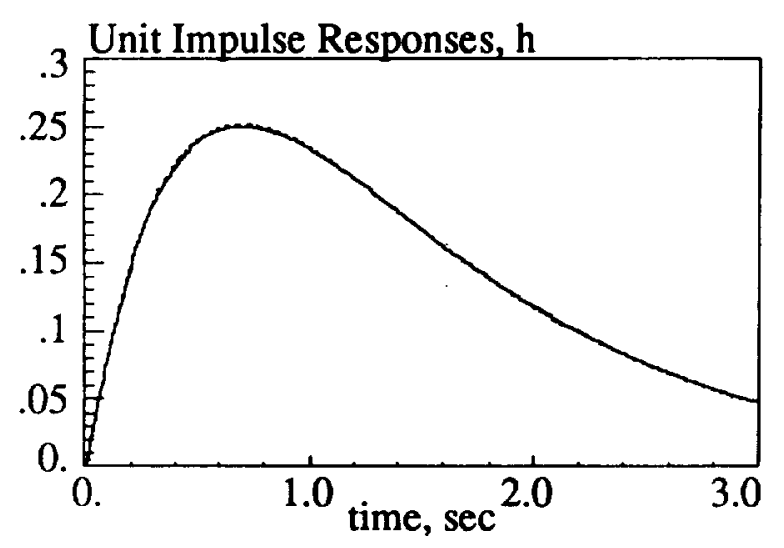

Figure 2 Comparison of the analytical and numericallyidentified linear unit impulse responses for the example problem.

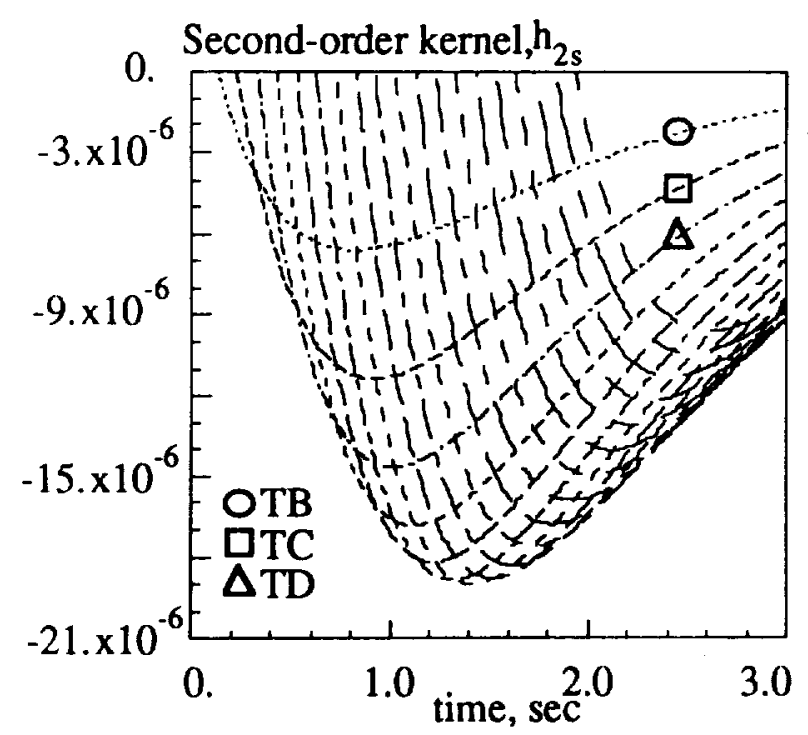

Figure 3 Numerically-identified second-order kernel at twenty values of time lag for the example problem.

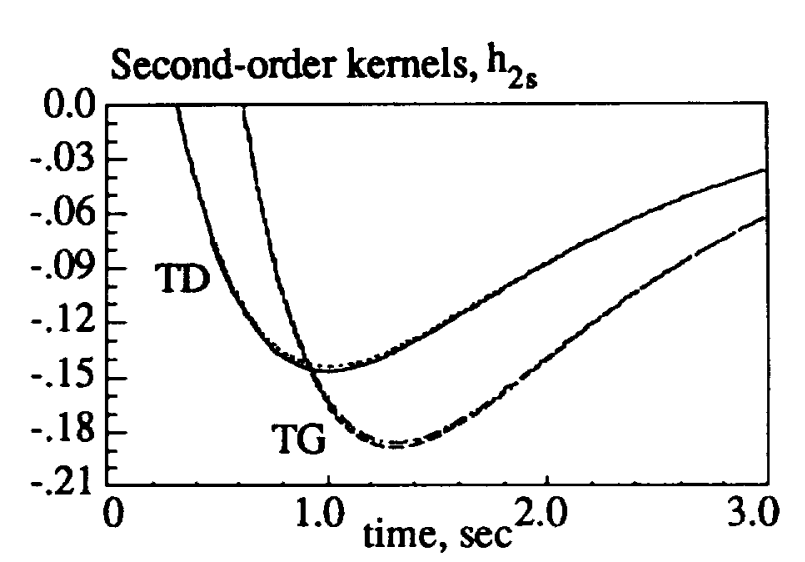

Figure 4 Comparison of analytical and numericallyidentified second-order kernels at two values of time lag for the example problem.

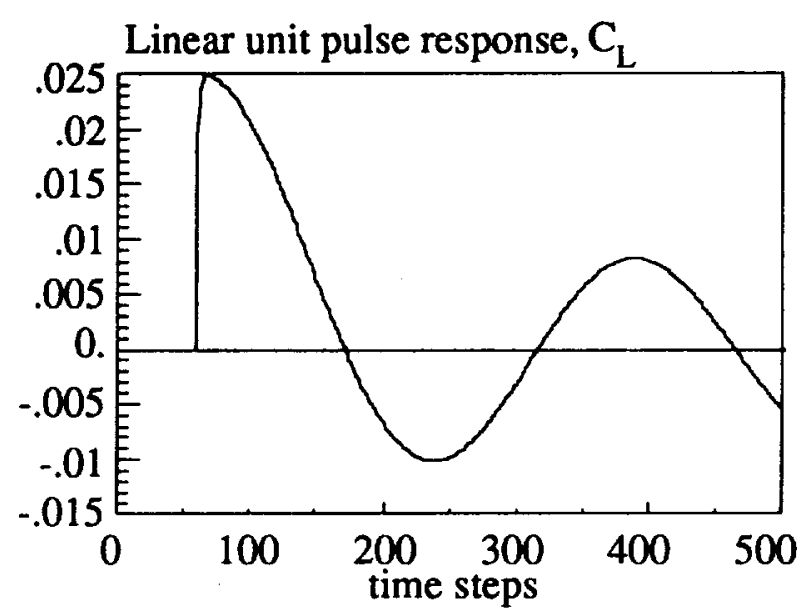

Figure 5 The lift-coefficient unit pulse response due to pitch at the quarter-chord for the linear (flat plate) aerodynamic solution at $\mathbf{M}=\mathbf{0 . 8 5}$.

$\mathrm{C}_{\mathrm{L}}$ and $\dot{\alpha}, \mathrm{rad} / \mathrm{sec}$

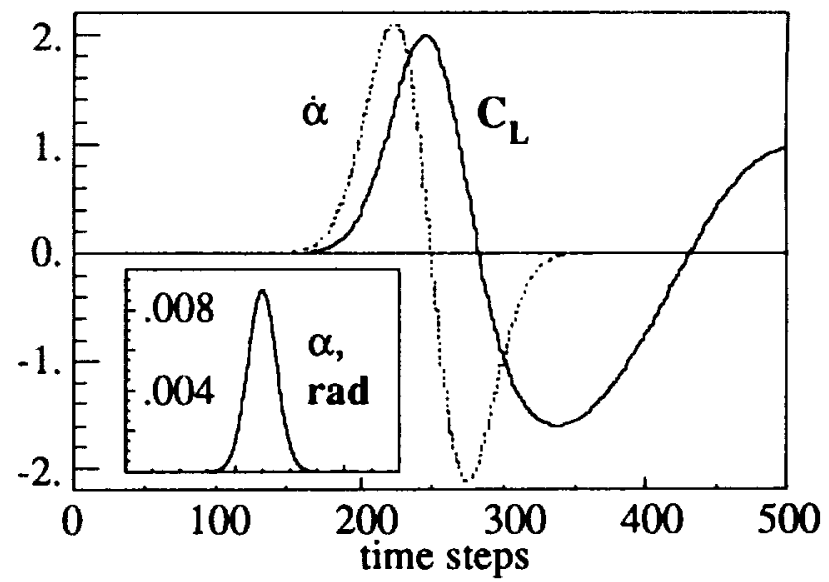

Figure 6 Lift-coefficient response to the pitching motion about the quarter-chord location described by the angle-ofattack (insel) and angle-of-attack rate functions for the linear acrodynamic (flat plate) solution at $\mathrm{M}=\mathbf{0 . 8 5}$. 


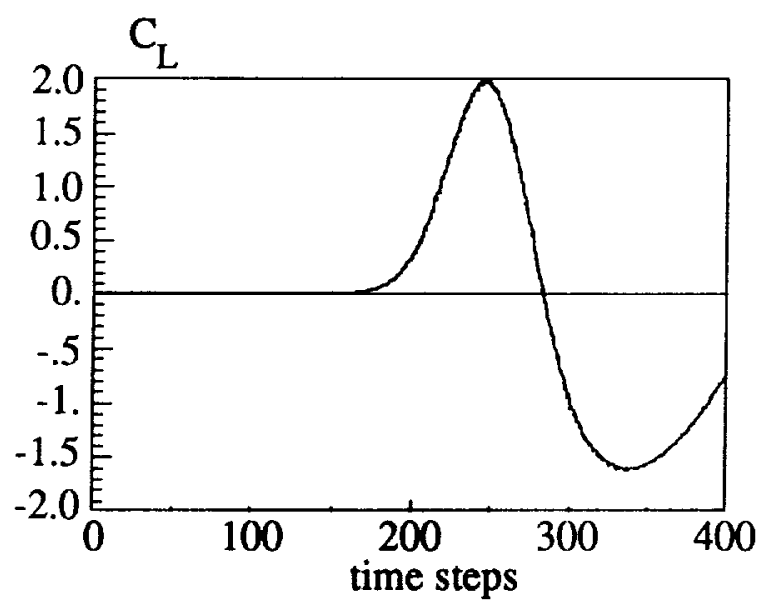

Figure 7 Comparison of lift-coefficient responses, due to the exponential pitch pulse shown in fig. 6 , for the CAP-TSD solution and the convolution of Pl (fig. 5) and the angle-of-attack rate (fig.6).

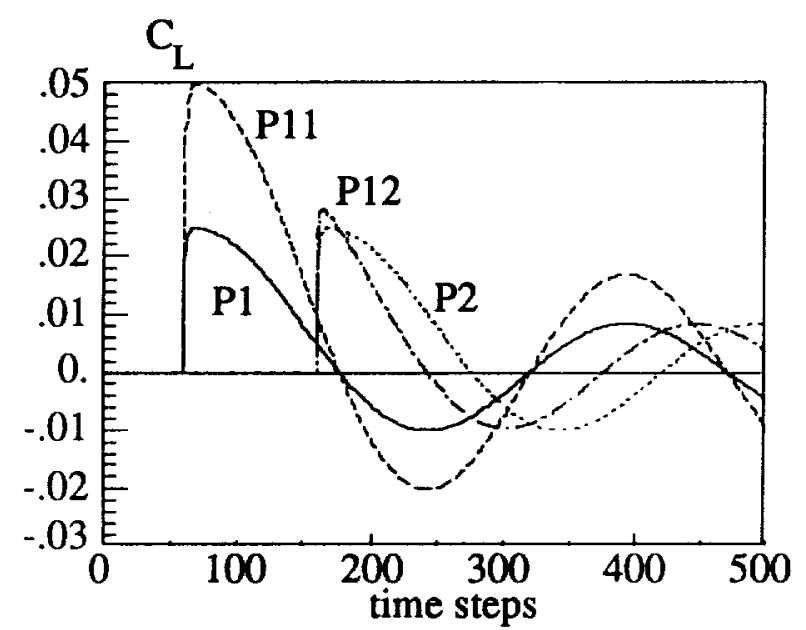

Figure 8 The nonlinear (thickness) lift-cocfficient unit pulse responses $\mathrm{P} 1, \mathrm{P} 2, \mathrm{P} 11$, and $\mathrm{P} 12$ due to pitch at the quarter-chord location for the NACA64A010 rectangular wing at $M=0.85$ and $\gamma=1.4$.

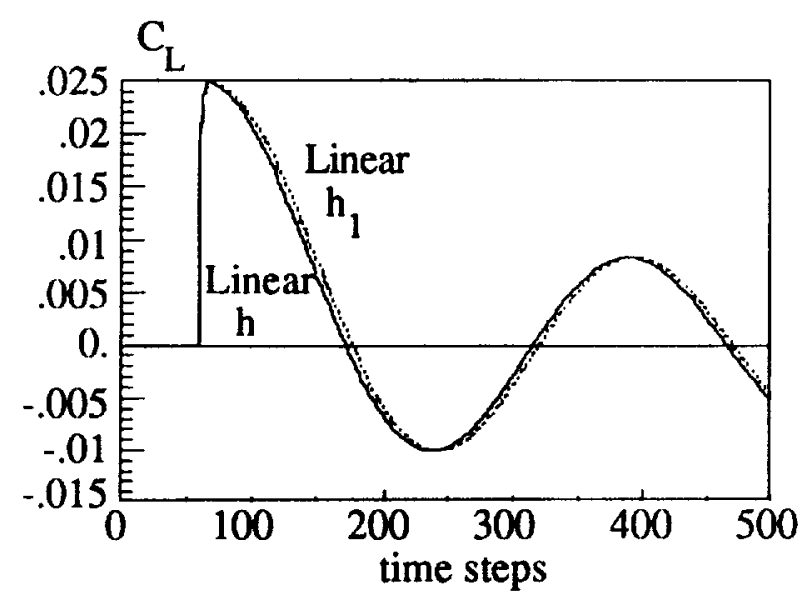

Figure 9 Comparison of the lift-coefficient responses due to pitch at the quarter-chord location for the linear (flat plate) case and the linear portion of the nonlinear (thickness) response case at $\mathrm{M}=0.85$ and $\gamma=1.4$.

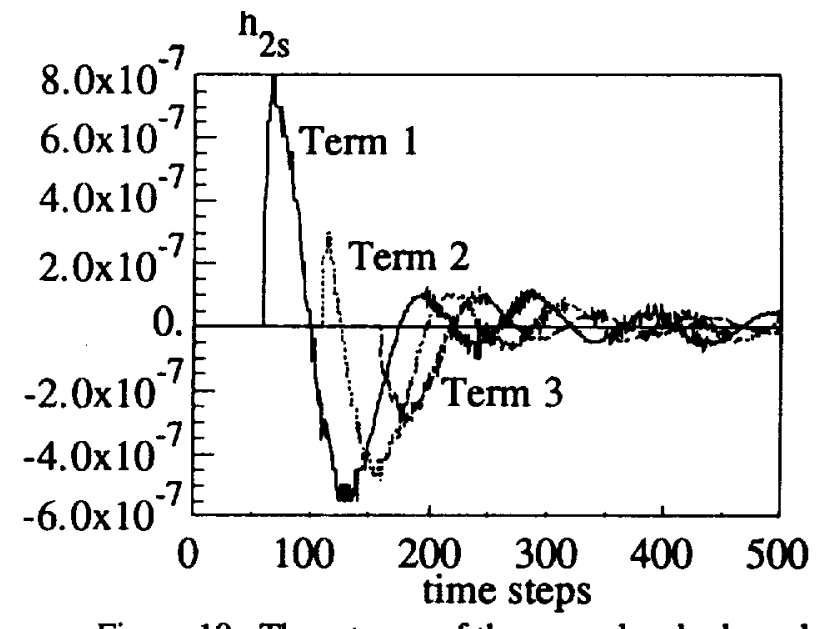

Figure 10 Three terms of the second-order kernel of lift coefficient due to pitch about the quarter-chord location for the NACA64A010 rectangular wing at $M=0.85$ and $\gamma=1.4$.

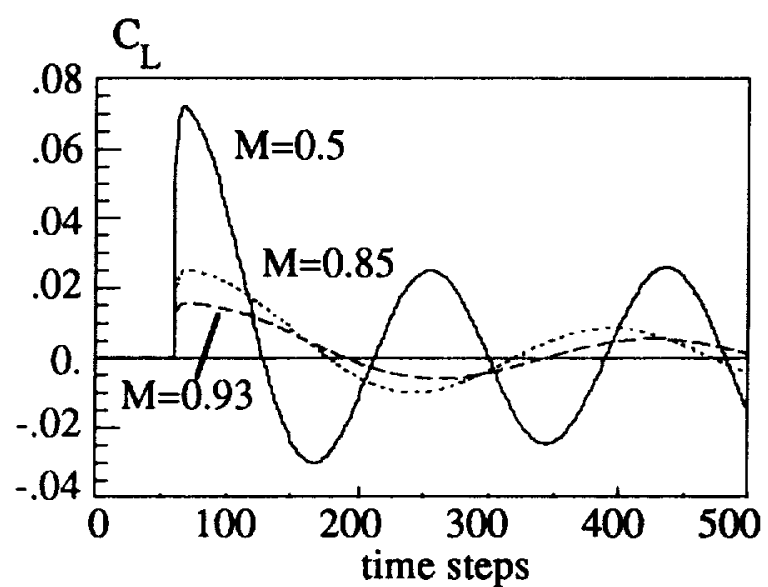

Figure 11 Comparison of nonlinear (thickness) liftcoefficient unit pulse responses due to pitch at the quarter-chord location for $\mathrm{M}=0.5, \mathrm{M}=0.85$, and $\mathrm{M}=0.93$ with $\gamma=1.4$.

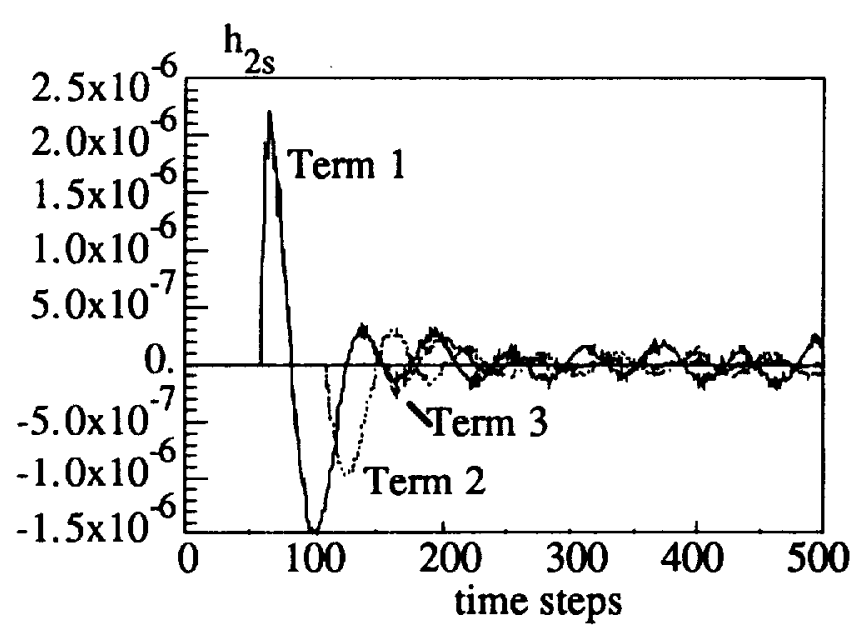

Figure 12 Three terms of the second-order kernel of lift coefficient due to pitch about the quarter-chord location for the NACA64A010 rectangular wing at $\mathrm{M}=0.50$ and $\gamma=1.4$. 


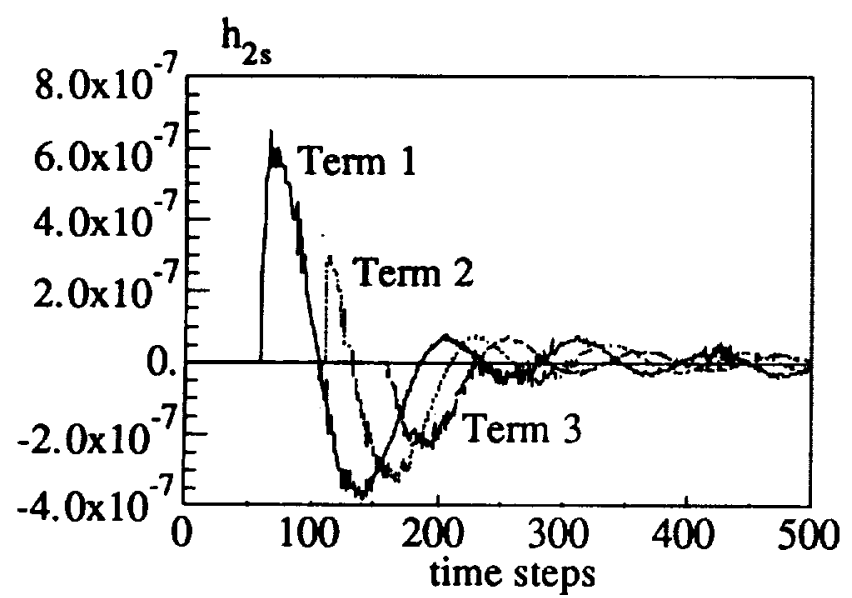

Figure 13 Three terms of the second-order kernel of lift coefficient due to pitch about the quarter-chord location for the NACA64A010 rectangular wing at $\mathrm{M}=0.93$ and $\gamma=1.4$.

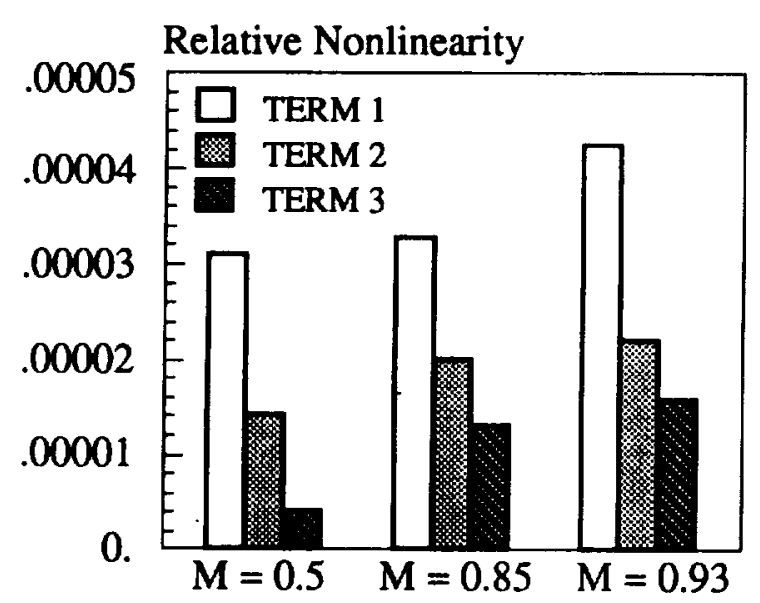

Figure 14 Relative nonlinearity for each of the three terms of the second-order kernel at $M=0.5$, $M=0.85$, and $M=0.93$.

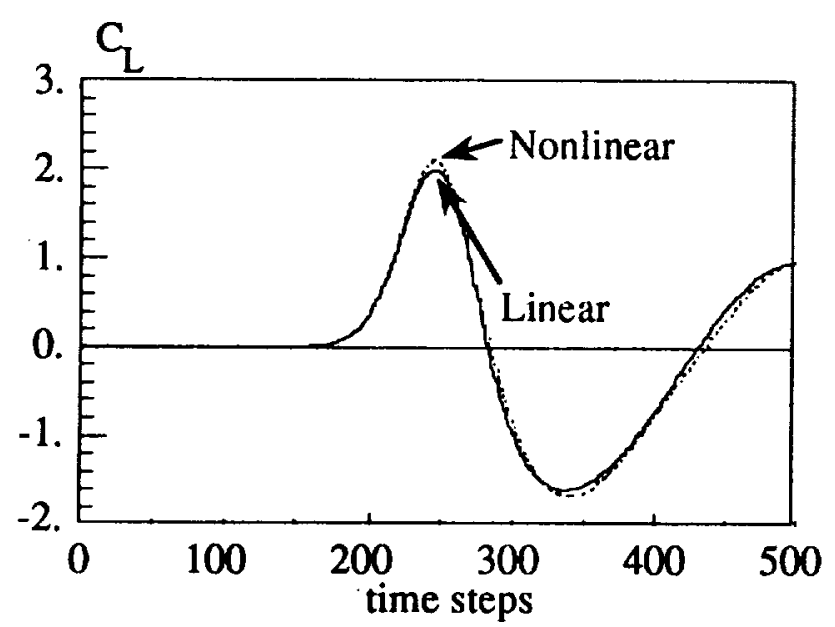

Figure 15 Comparison of linear (flat plate) and nonlinear (thickness) lift-coefficient responses due to the exponential pitch pulse shown in figure 6 at $M=0.85$ and $\gamma=1.4$ (for nonlinear case). 


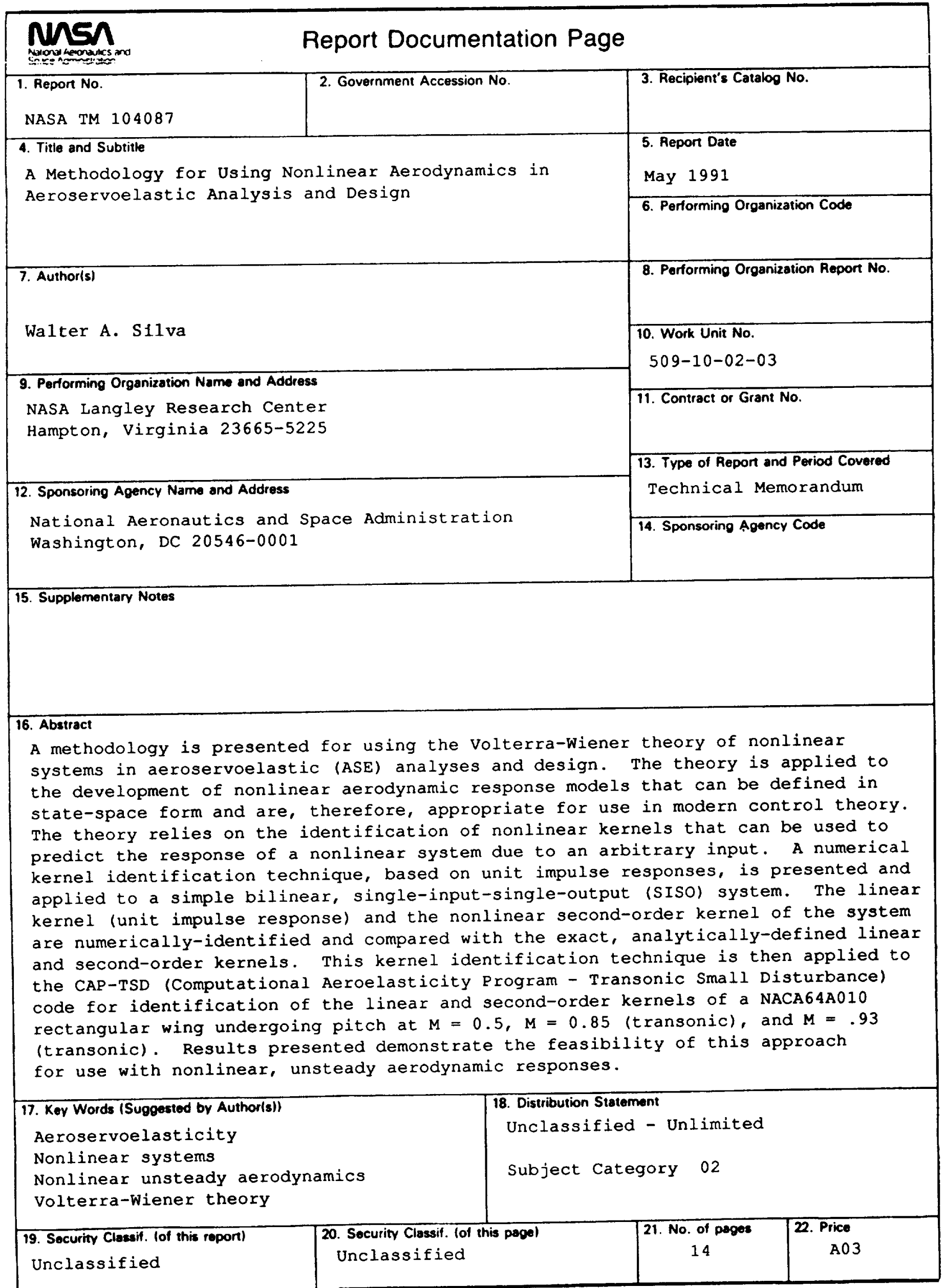




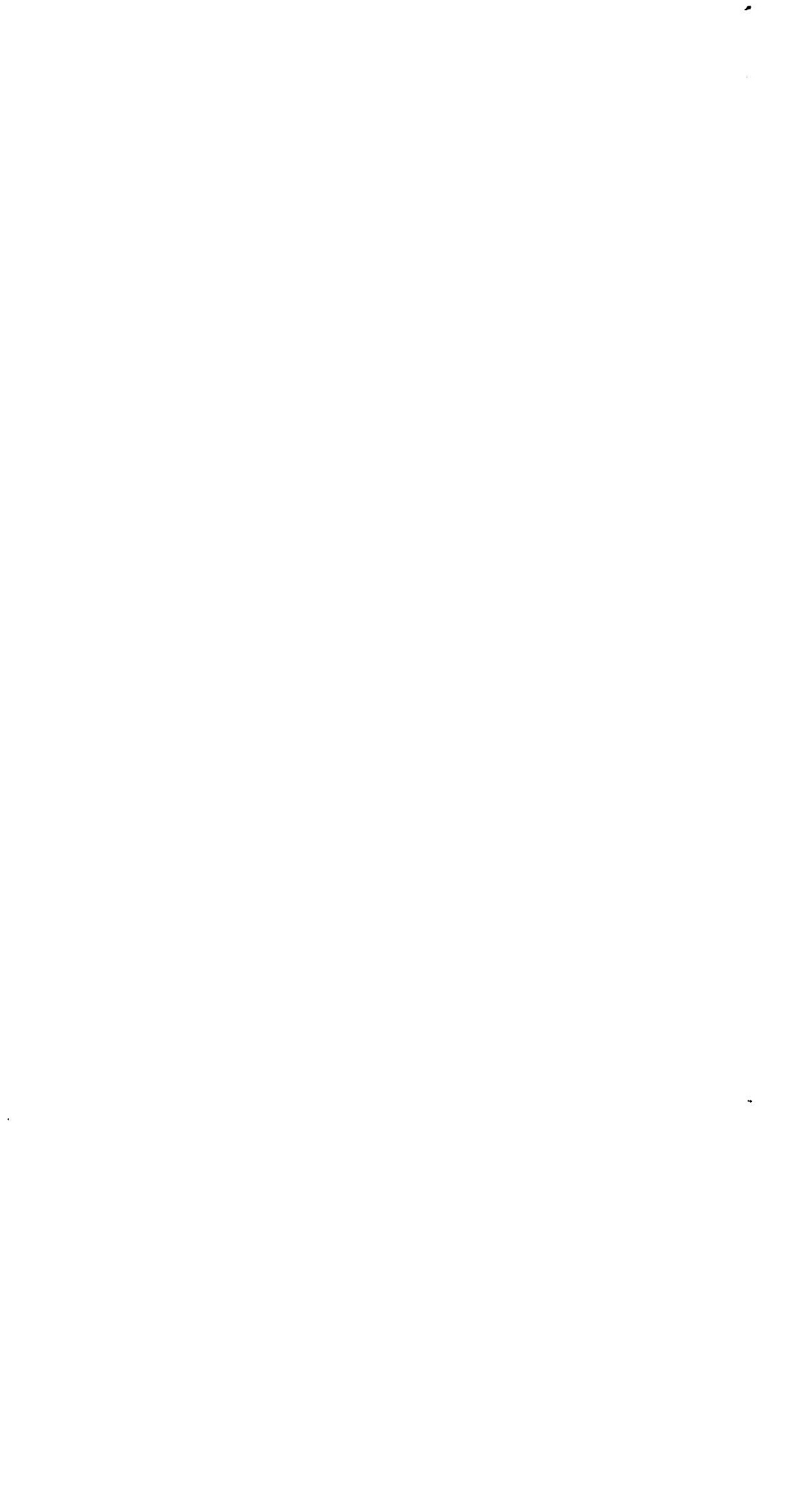

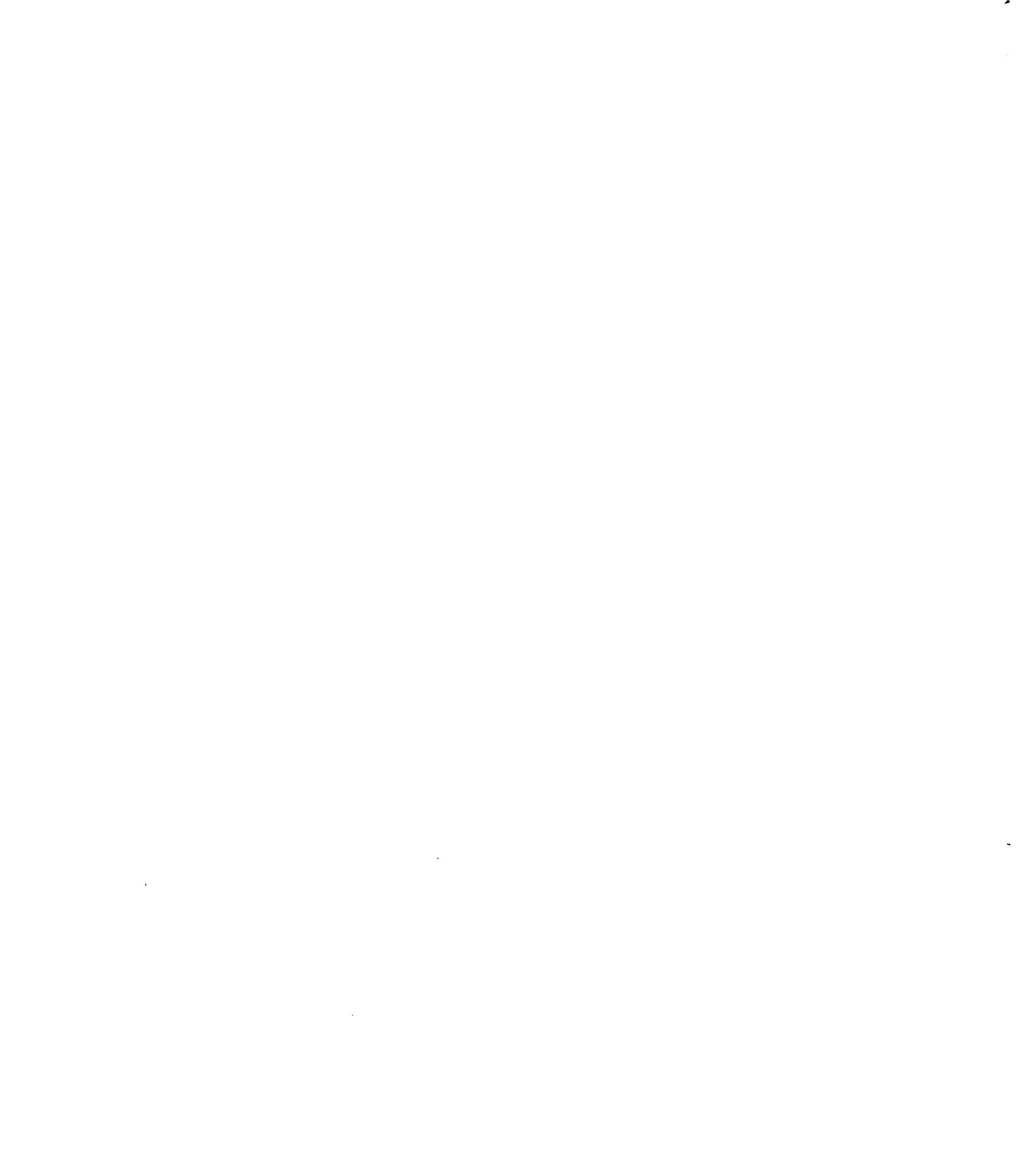

